\title{
Vein-related Trigeminal Neuralgia: How to Determine the Treatment Method of the Causative Vein: A Technical Note
}

\author{
Sho TsunODA, ${ }^{1}$ Tomohiro InOUE, ${ }^{1}$ Masafumi Segawa, ${ }^{1}$ and Atsuya AKABAnE ${ }^{1}$ \\ ${ }^{1}$ Department of Neurosurgery, NTT Medical Center Tokyo, Tokyo, Japan
}

\begin{abstract}
Trigeminal neuralgia (TN) is often caused by various vein forms. Herein, we report two cases of vein-related TN. In the first case, the vein of the cerebellopontine fissure (VCPF), along with the thickened surrounding arachnoid, clung to the trigeminal nerve. As the perfusion range of this vein was expected to be wide and its injury might cause extensive venous infarction, the anchor between the trigeminal nerve and the vein was carefully dissected and the vein was successfully transposed. In the second case, the involvement of the vein of the middle cerebellar peduncle (VMCP), which penetrates the nerve bundle of the trigeminal nerve, was suspected. Because vein transposition was impossible, the vein was transected at the nerve penetration site after confirming that there was sufficient collateral venous outflow. The superior petrosal vein and its tributaries are the primary causative veins of vein-related TN, and their contact patterns are infinite. Moreover, their perfusion range and the presence or absence of collateral venous outflow vary from case to case. Therefore, it is necessary to judge the perfusion range as well as collateral venous outflow based on preoperative images and intraoperative findings and to determine the appropriate treatment method for each case.
\end{abstract}

Keywords: trigeminal neuralgia, vein-related, petrosal vein, diagnostic imaging

\section{Introduction}

Trigeminal neuralgia (TN) is frequently caused by various vein forms, including venous compression alone, compression of both veins and arteries, and nerve entrapment due to thickened arachnoid around the petrosal veins. ${ }^{1-8)}$ During surgical treatment, sacrificing the causative vein is often unavoidable when the transposition is anatomically or technically difficult or if the offending vein, the so-called intraneural vein, that penetrates a band of nerve fibers. ${ }^{8)}$ Among the veins that comprise the petrosal vein complex, some can be cut without any adverse effects, ${ }^{3,8)}$ whereas in others, the cut may cause severe venous infarction and serious symptoms. ${ }^{2,9)}$ Nonetheless, no consensus has been established in determining whether safe transection is possible.

Received May 22, 2021; Accepted September 14, 2021

Copyright $@ 2021$ The Japan Neurosurgical Society This work is licensed under a Creative Commons AttributionNonCommercial-NoDerivatives International License.
In this report, we present two cases of vein-related TN with an extremely rare venous anatomy and discuss the treatment method criteria for vein-related $\mathrm{TN}$ based on the venous anatomy around the trigeminal nerve.

\section{Case Report}

\section{Case 1}

A 53-year-old woman complained of stinging pain around her right nasal wing and mandible while washing her face. In addition to carbamazepine (200 mg/day), the mental nerve, infraorbital nerve, and Gasserian ganglion block were repeated; however, her symptoms gradually deteriorated. She constantly felt pain of 3-4 on the numerical rating scale (NRS), and a pain score of 10 (on NRS) with lacrimation persisted for several days during the pain attack; thus, she requested surgical treatment 8 years after the first visit.

On preoperative T2-weighted volume isotropic turbo spin-echo acquisition (T2 VISTA) magnetic resonance imaging (MRI), the vein of the cerebellopontine fissure 

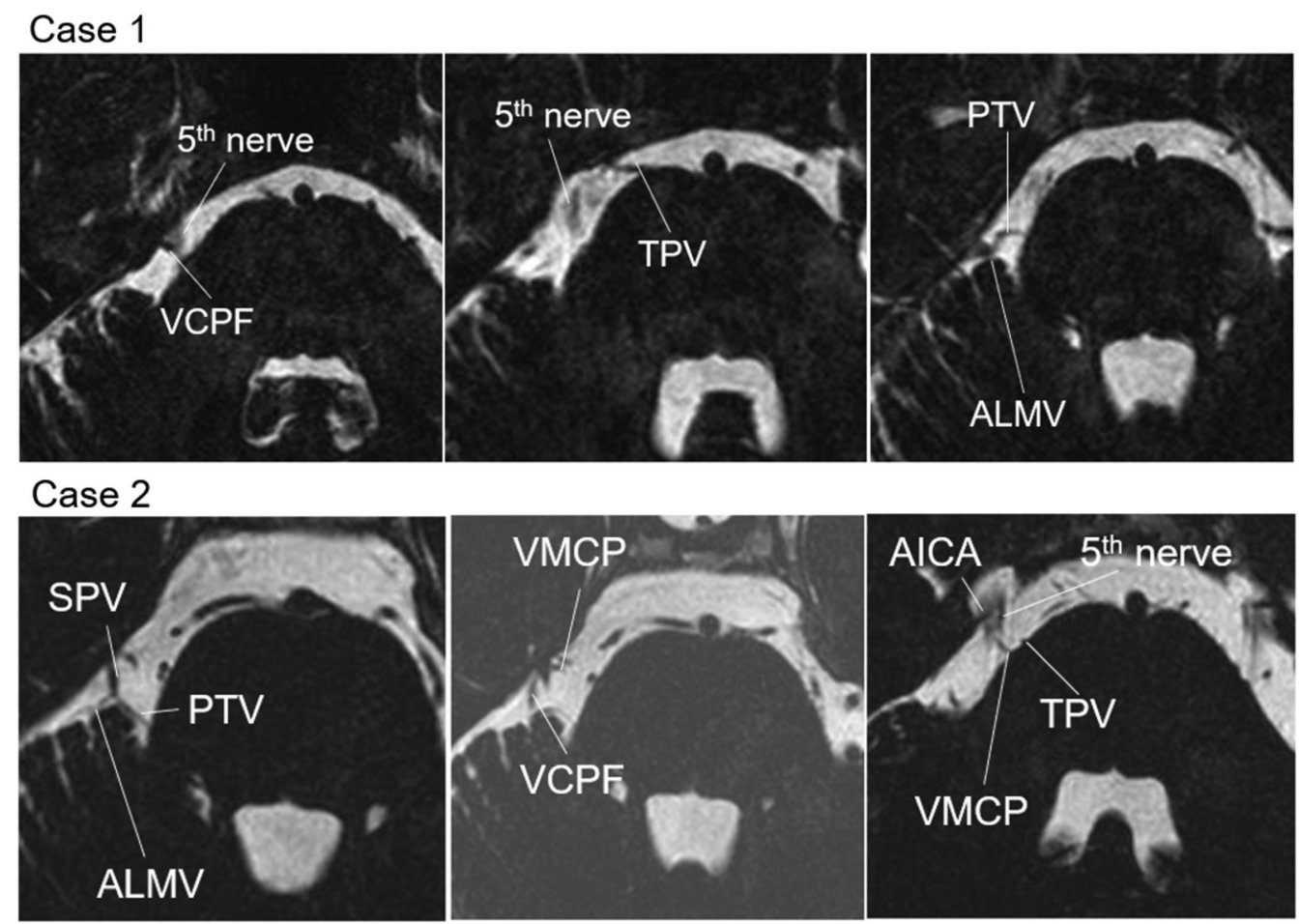

Fig. 1 Preoperative T2 VISTA MRI of two cases. In Case 1, the cause was nerve compression due to VCPF. In Case 2, the main cause was considered AICA near the Meckel cave and VMCP near the REZ of the trigeminal nerve. AICA: anterior inferior cerebellar artery, ALMV: anterior lateral marginal vein, MRI: magnetic resonance imaging, PTV: pontotrigeminal vein, REZ: root entry zone, SPV: superior petrosal vein, TPV: transverse pontine vein, VCPF: vein of the cerebellopontine fissure, VMCP: vein of the middle cerebellar peduncle.

(VCPF) contacted the trigeminal nerve on the dorsal side near the Meckel cave (Fig. 1).

Three bridging veins were observed intraoperatively. In the first, the anterior lateral marginal vein (ALMV), which received blood flow from the hemispheric veins, and the pontotrigeminal vein (PTV), which rose from the dorsolateral part of the brainstem, merged and flowed to the superior petrosal sinus (SPS) in the shallow layer on the cranial side of the trigeminal nerve (Fig. 2, SPV 1). In the second, the transverse pontine vein (TPV) flowed directly into the inferior petrosal sinus (IPS), deeper than the trigeminal nerve (Fig. 2, SPV 2). In the third, VCPF that arose from the caudal side of the trigeminal nerve flowed to SPS near the porus trigeminus (Fig. 2, SPV 3) and along with the thickened surrounding arachnoid, clung to the trigeminal nerve on the dorsocaudal side and flexed the nerve (Fig. 2A, blue arrowheads).

Each vein flowed to SPS or IPS as an independent bridging vein, and VCPF had no collateral venous outflow; hence, sacrificing it was expected to result in extensive postoperative venous infarction (Fig. 2B, blue circle). Therefore, although VCPF strongly adhered to the trigeminal nerve, the anchor between the nerve and the vein was meticulously released and VCPF was transposed to the dorsal petrous dura (Fig. 2A, black arrows).

Although localized pain (approximately NRS 2) around the right nasal wing persisted during the non-ictal period, the patient's pain attack disappeared from postoperative day (POD) 1. Pain in the non-ictal period was controlled by administering carbamazepine (200 mg/day), and the patient was satisfied.

\section{Case 2}

A 51-year-old woman developed unbearable pain on the lower right molars. She was unable to brush her teeth because of pain from a decayed tooth, resulting in tooth extraction and sequestrectomy. Her symptoms did not improve, and carbamazepine was discontinued because of pruritus. Thereafter, nonsteroidal anti-inflammatory drugs (NSAIDs) were used during the pain attack but with limited effects. The pain gradually worsened to an NRS of 10, and she requested surgical treatment 13 years after the initial visit.

On preoperative T2 VISTA, the main cause was considered the anterior inferior cerebellar artery contact (AICA) near the Meckel cave, and involvement 

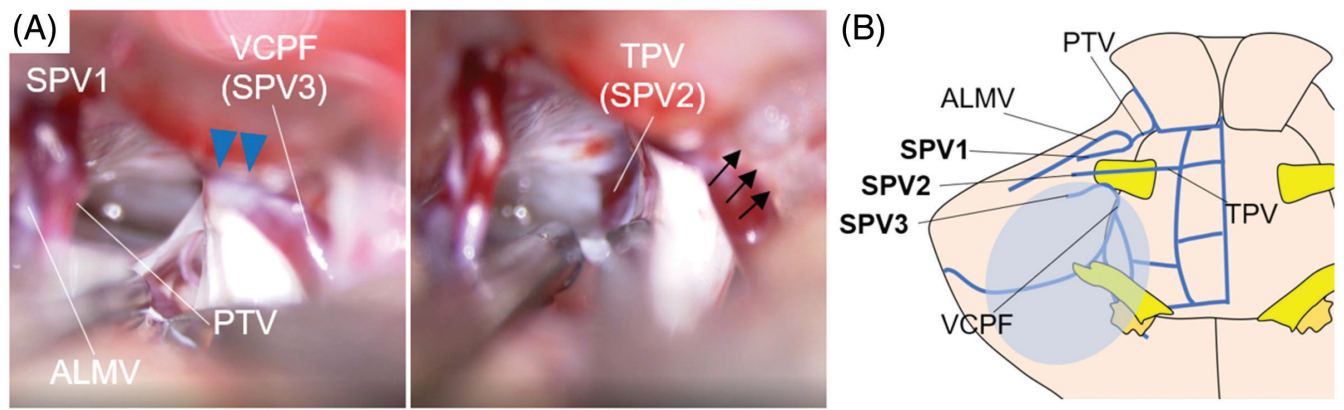

Fig. 2 (A) Intraoperative findings of Case 1. VCPF, with the thickened surrounding arachnoid, clung to the trigeminal nerve from the dorsocaudal side and flexed the nerve (blue arrowheads). The vein was transposed posteriorly and adhered to the petrous dura (black arrows). (B) A schema of venous anatomy in Case 1. VCPF was expected to receive extensive venous perfusion (blue circle). ALMV: anterior lateral marginal vein, PTV: pontotrigeminal vein, SPV: superior petrosal vein, T2 VISTA: T2-weighted volume isotropic turbo spin-echo acquisition, TPV: transverse pontine vein, VCPF: vein of the cerebellopontine fissure.
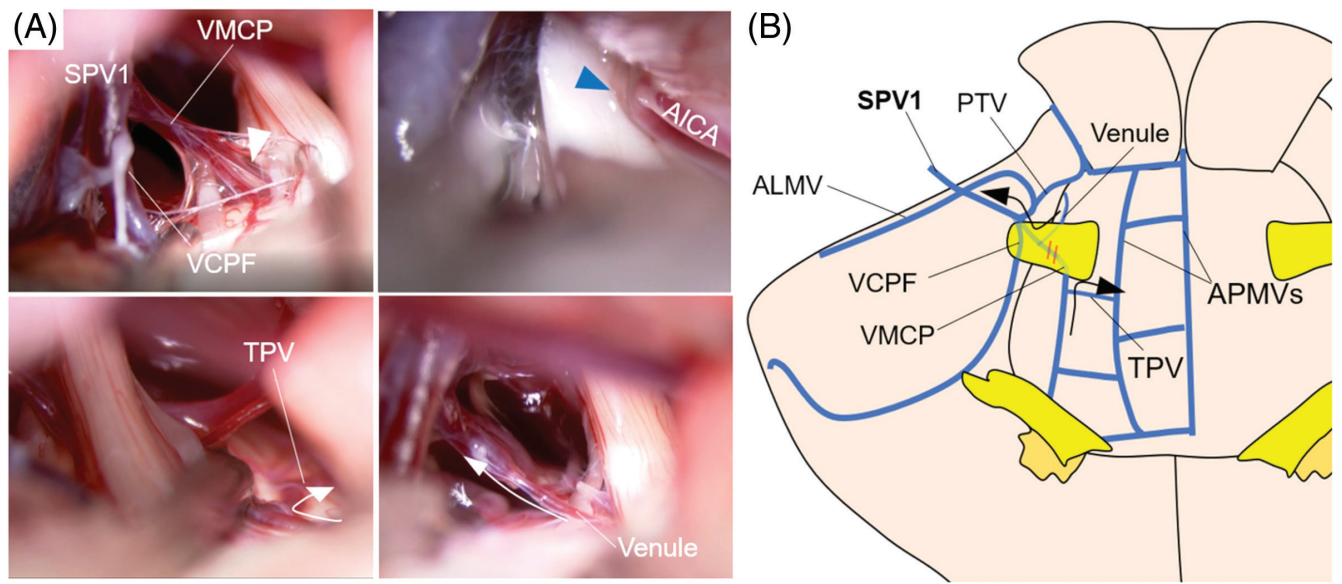

Fig. 3 (A) Intraoperative findings of Case 2. VMCP penetrated the trigeminal nerve (white arrowhead) and AICA (blue arrowhead) compressed the nerve. Assuming VMCP on the caudal side outflow into APMV via TPV and the venule on the cranial side outflow into the main trunk of VMCP, respectively (white arrow), VMCP was transected at the nerve penetration site. (B) A schema of venous anatomy in Case 2. ALMV, VCPF, VMCP, and PTV were merged and drained into SPS as a single bridging vein (SPV 1). Collateral venous outflow of each VMCP and venule was expected (black arrows), even if VMCP was transected at the nerve penetration part (orange double line). AICA: anterior inferior cerebellar artery, ALMV: anterior lateral marginal vein, APMV: anterior pontomesencephalic vein, PTV: pontotrigeminal vein, SPV: superior petrosal vein, TPV: transverse pontine vein, VCPF: vein of the cerebellopontine fissure, VMCP: vein of the middle cerebellar peduncle.

of the vein of the middle cerebellar peduncle (VMCP) around the root entry zone (REZ) was also suspected (Fig. 1).

Intraoperatively, AICA that bent the trigeminal nerve near the porus trigeminus was first posteriorly transposed (Fig. 3A, blue arrow), and the venous anatomy around the trigeminal nerve was properly inspected. Then, ALMV, PTV, VCPF, and VMCP merged and flowed to SPS as a single bridging vein (Fig. 3, SPV 1), and among them, VMCP, which penetrated the nerve bundle of the trigeminal nerve near REZ, seemed to compress the sensory root from the ventral side (Fig. 3A, white arrowhead). It was difficult to judge whether VMCP was actually associated with TN, but the possibility was not negligible.

This vein received blood flow of TPV from the ventrocaudal side of the trigeminal nerve and venule at the dorsolateral brain stem on the cranial side, respectively. Due to the presence of thick VCPF, the perfusion range of VMCP was not expected to be wide. Therefore, assuming VMCP on the caudal side of the trigeminal nerve outflow into the ventral brainstem draining root, such as the medial and lateral anterior pontomesencephalic veins (APMVs), 
via TPV and the venule on the cranial side of the trigeminal nerve outflow into the main trunk of VMCP (Fig. 3A, white arrow) (Fig. 3B, black arrow), VMCP was transected at the nerve penetration site.

The patient's symptoms immediately disappeared from POD 1, and NSAID use became unnecessary.

The surgical videos of the above two cases are shown in Video 1 (available Online).

\section{Discussion}

Vein-related TN is primarily caused by SPV tributary veins. ${ }^{4,6,10)}$ These tributary veins flow into SPS via 1-3 SPVs, and SPV and SPS conference varies depending on the complexity of the petrosal vein around the trigeminal nerve. Hence, the contact patterns of bridging veins and their tributaries can be infinite in both number and direction. ${ }^{1,7,11,12)}$

Matsushima et al. classified veins around the trigeminal nerve into the following four groups: group 1: anterior pontomesencephalic draining; group 2: posterior mesencephalic draining; group 3: tentorial cerebellar surface draining; and group 4: petrosal fissure draining. ${ }^{11)}$ Preoperative imaging should be used to assess the group to which the causative vein belongs, and treatment should be decided after identifying its perfusion range and collateral venous outflow.

If the causative vein has wide perfusion range and scarce collateral venous outflow, its transection is contraindicated, and transposition of the vein is required. It is known that mechanical nerve injury occurs by reckless manipulation around a nerve and worsens facial numbness postoperatively ${ }^{2,8}$; hence, an advanced technique is needed to dissect the anchoring arachnoid between the vein and the nerve while pulling the vein with the proper tension without stimulating the nerve. Furthermore, regarding the transposition method, the venous obstruction risk is higher when pulling the causative vein by the Teflon sling due to the thin wall and low elasticity of the vein. ${ }^{3)}$ Therefore, it is desirable to pull the causative vein together with the surrounding arachnoid and stuck the arachnoid and petrosal dura by the Birdlime technique. ${ }^{13)}$ In our Case 1, slight symptoms persisted after treatment; however, it is unknown whether this was due to nerve injury associated with surgical manipulation or repeated nerve block. Thus, the treatment of venous TN is far more difficult than that of arterial TN in terms of curability.

If the transposition is anatomically or technically difficult, sacrificing the causative vein is unavoidable. Regarding the intraneural vein, there is no consensus on the safety of its transection. ${ }^{2,8}$ In general, the veins belonging to group 4 in Matsushima's classification should not be cut due to its wide perfusion range, but they can be reportedly cut when obviously thinner than the petrosal veins. ${ }^{3,11)}$ The risk of postoperative problem due to transection of TPV, which has sufficient collateral outflow anastomosing with the Galenic draining system via APMV is considered to be low. ${ }^{14,15)}$ By contrast, TPV congestion has also been reported to cause broad range venous infarction at the dorsolateral midbrain and superior cerebellar peduncle, ${ }^{9)}$ leading to serious symptoms. As for the other veins, there was no noticeable report. From the above, the risk of venous infarction after the transection of a causative vein cannot completely be predicted; thus, it is important to carefully judge whether transection is really necessary. Furthermore, when vein transection is unavoidable, it may be useful to temporarily clamp the causative vein and then observe changes in the auditory brainstem response and/or the venous congestion on indocyanine green (ICG) video angiography as reference findings. ${ }^{16-18)}$ Case 2 may have benefited from more detailed safety confirmation, including intraoperative ICG video angiography, prior to vein transection.

\section{Conclusion}

When treating vein-related TN surgically, even with the unpredictable risk of postoperative venous infarction, venous infarction of the brainstem can lead to serious symptoms even in small areas. Therefore, careful treatment selection of the causative vein should be determined on an individual basis.

\section{Conflict of Interest Disclosure}

On behalf of all authors, the corresponding author states that there is no conflict of interest.

\section{Supplemental Video}

This video clip demonstrates the actual surgical procedures performed in the two cases of veinrelated $\mathrm{TN}$.

\section{References}

1) Dumot C, Sindou M: Trigeminal neuralgia due to neurovascular conflicts from venous origin: an anatomical-surgical study (consecutive series of 124 operated cases). Acta Neurochir (Wien) 157: 455-466, 2015

2) Helbig GM, Callahan JD, Cohen-Gadol AA: Variant intraneural vein-trigeminal nerve relationships: an observation during microvascular decompression surgery for trigeminal neuralgia. Neurosurgery 65: 958-961; discussion 961, 2009 
3) Inoue T, Hirai H, Shima A, Suzuki F, Fukushima T, Matsuda M: Diagnosis and management for trigeminal neuralgia caused solely by venous compression. Acta Neurochir (Wien) 159: 681-688, 2017

4) Kimura T, Sako K, Tohyama Y, Yonemasu Y: Trigeminal neuralgia caused by compression from petrosal vein transfixing the nerve. Acta Neurochir (Wien) 141: 437-438, 1999

5) Lee SH, Levy EI, Scarrow AM, Kassam A, Jannetta PJ: Recurrent trigeminal neuralgia attributable to veins after microvascular decompression. Neurosurgery 46: 356-361; discussion 361-362, 2000

6) Matsushima T, Huynh-Le P, Miyazono M: Trigeminal neuralgia caused by venous compression. Neurosurgery 55: 334-337; discussion 338-339, 2004

7) Wu M, Fu X, Ji Y, et al.: Microvascular decompression for classical trigeminal neuralgia caused by venous compression: novel anatomic classifications and surgical strategy. World Neurosurg 113: e707-e713, 2018

8) Zheng $\mathrm{X}$, Feng B, Hong W, et al.: Management of intraneural vessels during microvascular decompression surgery for trigeminal neuralgia. World Neurosurg 77: 771-774, 2012

9) Liebelt BD, Barber SM, Desai VR, et al.: Superior petrosal vein sacrifice during microvascular decompression: perioperative complication rates and comparison with venous preservation. World Neurosurg 104: 788-794, 2017

10) Rhoton AL: The cerebellopontine angle and posterior fossa cranial nerves by the retrosigmoid approach. Neurosurgery 47: S93-129, 2000

11) Matsushima K, Matsushima T, Kuga Y, et al.: Classification of the superior petrosal veins and sinus based on drainage pattern. Oper Neurosurg 10: 357-367, 2014
12) Basamh M, Sinning N, Kehler U: Individual variations of the superior petrosal vein complex and their microsurgical relevance in 50 cases of trigeminal microvascular decompression. Acta Neurochir (Wien) 162: 197-209, 2020

13) Otani N, Toyooka T, Fujii K, et al.: "Birdlime” technique using TachoSil tissue sealing sheet soaked with fibrin glue for sutureless vessel transposition in microvascular decompression: operative technique and nuances. J Neurosurg 128: 1522-1529, 2018

14) Cai M, Zhang XF, Qiao HH, et al.: Susceptibilityweighted imaging of the venous networks around the brain stem. Neuroradiology 57: 163-169, 2015

15) Matsushima T, Rhoton AL, de Oliveira E, Peace D: Microsurgical anatomy of the veins of the posterior fossa. J Neurosurg 59: 63-105, 1983

16) Ferroli P, Nakaji P, Acerbi F, Albanese E, Broggi G: Indocyanine green (ICG) temporary clipping test to assess collateral circulation before venous sacrifice. World Neurosurg 75: 122-125, 2011

17) Strauss C, Neu M, Bischoff B, Romstöck J: Clinical and neurophysiological observations after superior petrosal vein obstruction during surgery of the cerebellopontine angle: case report. Neurosurgery 48: 1157-1161, 2001

18) Zhong J, Li ST, Xu SQ, Wan L, Wang X: Management of petrosal veins during microvascular decompression for trigeminal neuralgia. Neurol Res 30: 697700, 2008

Corresponding author: Sho Tsunoda, MD

Department of Neurosurgery, NTT Medical Center Tokyo, 5-9-22 Higashigotanda, Shinagawa-ku, Tokyo 141-0022, Japan.

e-mail: s.tsunoda1024@gmail.com 